Evaluar.

2003, $n^{\circ} 3$ (julio)
Laboratorio de Evaluación Psicológica y Educativa. Facultad de Psicología Universidad Nacional de Córdoba (Argentina).ISSN N ${ }^{\circ} 1515$ - 1867

\title{
Cuestionario de Intereses Profesionales Revisado. (CIP-R). Análisis de sus Propiedades Psicométricas
}

\author{
Hermelinda Fogliatto *, Edgardo Pérez *, Fabián Olaz * \& Luis Parodi * \\ *Lab. de Evaluación Psicológica y Educativa. Facultad de Psicología. Universidad Nacional de Córdoba
}

Resumen En el presente trabajo se informa acerca de la revisión y actualización del Cuestionario de Intereses Profesionales (Fogliatto, 1989; 1993), un instrumento elaborado en la Argentina con la finalidad de evaluar preferencias asociadas a carreras. Se obtuvieron evidencias de consistencia interna, estabilidad, validez conceptual y de criterio, con resultados satisfactorios para todas las escalas. El Cuestionario de Intereses Profesionales Revisado (CIP-R) se integrará como un módulo específico en la nueva versión del Sistema de Orientación Vocacional Informatizado (SOVI) (Fogliatto \& Pérez, 1997; 2001; en prensa). Este software de orientación asistida por computadora incluye evaluación de Intereses, de Autoeficacia para Inteligencias Múltiples (Pérez, 2001) y un módulo renovado de información sobre carreras contemplando hipervínculos a sitios web de Universidades del país.

Palabras Clave: Intereses Vocacionales - Tests Psicológicos - Orientación Vocacional

\section{Introducción}

Los intereses vocacionales han sido definidos como: "Patrones de gustos, indiferencias y aversiones respecto a actividades relacionadas con carreras y ocupaciones" (Lent, Brown \& Hackett, 1994). La problemática de los intereses ha sido exhaustivamente examinada por los investigadores del comportamiento vocacional. Se estima que un conocimiento adecuado de esta dimensión de la motivación humana permite predecir el monto de satisfacción que una persona experimentará en el desempeño de una ocupación (Barak, 1981).

Los intereses se relacionan también significativamente con la estabilidad y compromiso de los individuos en sus carreras y ocupaciones (Super, 1967). Otros investigadores han comparado el peso relativo de la variable "intereses" en relación con 
otras variables psicológicas (habilidades, rasgos de personalidad) en la elección de carrera, verificando que los intereses reciben gran consideración por parte de la persona en situaciones de decisión vocacional (Strong, 1951; Barak, 1981;Holland, 1997).

Los inventarios de intereses son, quizá, los instrumentos más populares en evaluación del comportamiento vocacional, según se desprende de encuestas realizadas en Estados Unidos, donde instrumentos como el Strong Campbell Interest Inventory son empleados por casi el $90 \%$ de los orientadores (Watkins, 1994). Los cuestionarios o inventarios de intereses han sido caracterizados como serie de ítems en los que se solicita a los individuos indicar sus preferencias vocacionales y que poseen un valor numérico que permite obtener una puntuación final que representa un perfil o pauta de intereses (Super, 1967; Cronbach, 1998; Roe, 1972).

En cuanto a su utilidad, los investigadores coinciden en señalar que deben aplicarse con fines de: seleccionar metas vocacionales, confirmar elecciones previas, descubrir campos de actividad laboral, incrementar el autoconocimiento y encontrar ocupaciones que proporcionen satisfacción (Cronbach, 1998; Rivas, 1988; Hood \& Jonhson, 2002). Es claro que los inventarios de intereses poco nos dicen respecto al éxito académico u ocupacional que podrá alcanzar una persona, pero nos ayudan a identificar carreras u ocupaciones donde el sujeto puede encontrar satisfacción.

Debe evitarse la práctica profesional de usar los inventarios de intereses para orientar de manera específica a los estudiantes, puesto que éstos necesitan considerar, en el proceso de toma de decisiones de carrera, variables igualmente relevantes y, además, reunir experiencia exploratoria sobre carreras y ocupaciones (Hood \& Johnson, 2002). En general, se recomienda confiar en los resultados de los inventarios de intereses, con finalidades de orientación vocacional a partir de los 15-17 años, aproximadamente (Super, 1967, Care, 1996). Strong (1951) verificó que las puntuaciones de intereses son realmente estables a partir de esa edad.

En este trabajo nos propusimos revisar y actualizar el Cuestionario de Intereses Profesionales (Fogliatto, 1991; 1993), un instrumento local de evaluación de preferencias vocacionales asociadas a carreras.

El paradigma más influyente en el dominio de la medición de los intereses es el formulado por Holland (1997). La teoría de Holland ha sido descrita como un modelo de congruencia entre los intereses y habilidades de una persona por un lado y los 
factores inherentes a su ambiente (Furham, 1992). Según este modelo, existen seis tipos de personalidad: Realista, Investigador, Artista, Social, Emprendedor y Convencional (RIASEC), que a su vez determinan seis patrones de intereses y habilidades. El desarrollo de esta tipología vocacional depende de una serie compleja de acontecimientos familiares, orientaciones personales iniciales, preferencias ocupacionales e interacciones con contextos ambientales específicos. Los ambientes en los que viven y trabajan las personas pueden también caracterizarse de acuerdo a su semejanza con seis modelos que se corresponden con los seis tipos de personalidad anteriormente mencionados. Se han descrito otros modelos complementarios del modelo RIASEC, como el de Hogan (1983), quién argumenta que hay dos dimensiones subyacentes al modelo RIASEC: Sociabilidad y Conformidad. El polo positivo de Sociabilidad está igualmente vinculado a los tipos Social y Emprendedor de Holland y su polo opuesto es equidistante de los tipos Realista e Investigador. El polo positivo de Conformidad coincide exactamente con el tipo Convencional de Holland y su polo opuesto con el tipo Artista. Se ha expresado que el modelo de Hogan facilita la vinculación del paradigma de Holland con las teorías de la personalidad (Schneider et al., 1996). La figura 1 ilustra las interesantes relaciones entre las teorías de Holland y Hogan.

\section{Figura 1. Relaciones entre el modelo RIASEC de Holland y el Bidimensional de Hogan.}

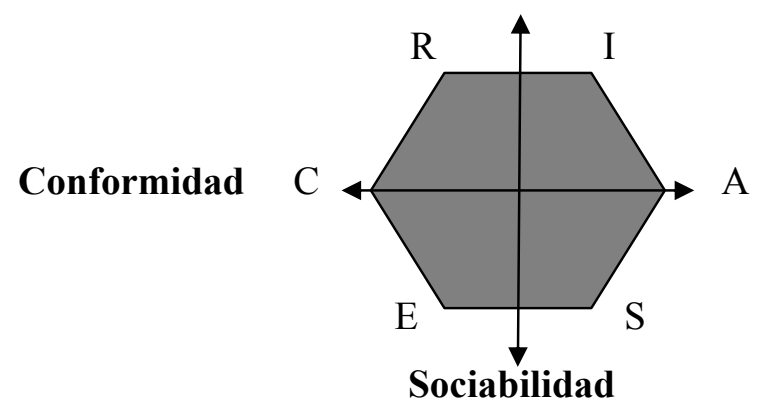

Los Inventarios de Intereses más populares son las escalas de Holland, el Inventario de Strong-Campbell y el Registro de Preferencias Kuder. Más allá de sus diferencias (Kuder obtuvo sus escalas por análisis factorial y emplea ítems de elección forzosa, Strong posee claves ocupacionales formadas por la comparación de personas 
satisfechas en una ocupación con respuestas representativas de todas las personas de la muestra), todos han adoptado el modelo RIASEC para interpretar los resultados lo cual permite una convergencia conceptual impensable en otros dominios de la psicología. Una iniciativa interesante es el Inventario Visual de Intereses Profesionales (Tetreau \& Trahan, 1986), desarrollado por investigadores canadienses y basado también en el modelo de Holland, pero que utiliza 80 fotografías en color ilustrando actividades laborales en lugar de ítems verbales, con el fin de atenuar los problemas de sesgo cultural.

No obstante, existen dificultades considerables cuando medidas elaboradas en otros contextos sociales se emplean de modo transcultural. Uno de los obstáculos más significativos en la traducción y adaptación de tests verbales son los problemas de lenguaje. En efecto, las traducciones libres pueden traicionar las intenciones originales del autor y las literales, por los problemas de equivalencia semántica y la diferente frecuencia de empleo de las palabras en lenguas diversas, no alcanzar a expresar precisamente los significados de los ítems en sus versiones originales (Cortada de Kohan, 1974).

También debe considerarse el papel de los factores culturales que pueden falsear de algún modo los resultados de tests cuando éstos se emplean en otros medios. Es frecuente encontrar en los inventarios de intereses, ítems que mencionan actividades que en sus culturas de origen poseen una popularidad que no tienen en otros contextos; jugar béisbol, por ejemplo, tiene diferente significación en USA que en otros países donde es un deporte poco practicado. Algunos ítems mencionan títulos ocupacionales que son familiares en el lugar de elaboración del inventario y, en cambio, resultan extraños para habitantes de otras regiones (Fogliatto, 1989).

En nuestro medio, Fogliatto et al. $(1973,1980)$ ratificaron estas conclusiones en un estudio empírico con estudiantes ingresantes a la Universidad Nacional de Córdoba, a quienes se les administró los Inventarios de Strong, Kuder y Holland. El funcionamiento de los ítems de estos instrumentos fueron analizados en tres niveles: semántico, de contenido y estadístico. Algunos de los principales resultados de esta investigación fueron los siguientes:

- Los instrumentos mencionados poseen una considerable cantidad de reactivos en áreas socialmente desprestigiadas en Argentina y han sido

Evaluar.

2003, $n^{\circ} 3$ (julio) 
diseñados para su empleo en la población general. Esto provocó rechazo por parte de los estudiantes de la muestra, hacia algunos ítems que se encuentran debajo de su nivel de aspiración vocacional.

- Existen numerosos ítems con problemas semánticos y aseveraciones que al ser traducidas han perdido su sentido original, lo cual crea problemas de comprensión del sentido del ítem.

- El análisis estadístico de los reactivos demostró que 137 reactivos del Kuder, 80 del Strong y 33 del Holland deberían ser revisados para su empleo en nuestra población pues no discriminan entre sujetos de carreras diferentes

Estos resultados indican con claridad los riesgos de emplear de modo acrítico inventarios de intereses construidos en otros contextos socioculturales. Fogliatto (1991) planteó la necesidad prioritaria de construir un cuestionario de intereses de características locales y más adecuadas a las preferencias, actividades educacionales, laborales así como lenguaje habitual de los jóvenes de nuestro medio. El programa de investigación dirigido por esta autora permitió crear el Cuestionario de Intereses Profesionales, versiones lápiz y papel (Fogliatto, 1991) y computarizado (Fogliatto, 1993). En este trabajo se presenta la revisión de este instrumento y los procesos de verificación de sus propiedades psicométricas esenciales. El Cuestionario de Intereses Profesionales Revisado (CIP-R) se integra como un módulo al Sistema de Orientación Vocacional Informatizado, en su versión 3 (Fogliatto \& Pérez, en prensa).

\section{Método}

\section{Instrumento}

El CIP-R comprende 114 reactivos que representan actividades académicas y ocupacionales relacionadas con carreras superiores, "Aprender estilos de pintura artística”, por ejemplo (Ver Anexo, Cuadernillo de Items). Los ítems se presentan en forma de aseveraciones a las cuales las personas evaluadas deben responder indicando su agrado, indiferencia o desagrado. El CIP-R permite obtener puntuaciones normativas en 15 escalas (Cálculo, Científica, Diseño, Tecnológica, Geoastronómica, Naturalista, Sanitaria, Asistencial, Jurídica, Económica, Comunicacional, Humanística, Artística, Musical y Lingüística) obtenidas por análisis factorial y relacionar cada perfil de intereses con opciones educativas. 


\section{Construcción}

Tomando como base fuentes de información profesiográfica tales como monografías profesionales, guías académicas de carreras y clasificaciones internacionales de ocupaciones, así como criterios de claridad semántica, corrección sintáctica y adecuación de los reactivos al nivel educativo y evolutivo de los adolescentes, se redactaron 450 ítems preliminares. Se confeccionaron tres cuestionarios preliminares que fueron administrados a una muestra de 1783 estudiantes de nivel medio y superior. El análisis factorial de las tres matrices de correlaciones dio como resultado 14, 13 y 17 factores que explicaban el 50\%, 50\% y 49\% de la varianza, respectivamente. A partir de los resultados de este estudio preliminar se elaboró un nuevo instrumento con los mejores 150 reactivos de las tres formas piloto. Los ítems de esta nueva versión del cuestionario fueron aquellos con cargas factoriales más elevadas y que aparecían solamente en un sólo factor. Esta nueva forma fue administrada a una muestra de 922 estudiantes del último año del nivel medio de educación. Empleando el método de extracción de Componentes Principales y el procedimiento de rotación Varimax se identificaron 16 factores que explicaban el 52,6\% de la varianza total. Se interpretaron 15 de estos factores dando lugar a las correspondientes escalas del cuestionario. Estos estudios así como los emprendidos para verificar la confiabilidad y validez de las escalas originales del CIP pueden consultarse en los manuales de las anteriores versiones, tanto lápiz y papel como basada en computadora (Fogliatto, 1991; 1993).

Una década después intentamos verificar la estabilidad y posibilidades de replicar la estructura factorial del CIP, además de investigar su relación con modelos racionales de medidas de intereses, construidos desde un marco teórico explícito y no puramente empírico. El CIP fue administrado a una muestra de 810 estudiantes de ambos sexos (mujeres 57,1\%, varones 42,9\%) con un rango de edad entre 16 y 20 años (media 17,48) y que cursaban el ciclo de especialización en sus distintas modalidades educativas (Producción de Bienes y Servicios, Comunicación, Arte y Diseño, Ciencias Sociales, Ciencias Naturales, Economía y Gestión de las Organizaciones). Se empleó el Método de Extracción de Componentes Principales y de Rotación Varimax. Se extrajeron 18 factores con Eigenvalues mayores que 1 y que explicaron el 63,04\% de la varianza. Se tomaron criterios de selección complementarios, tales como considerar la información aportada por el Scree Test así como el porcentaje de varianza explicada por

Evaluar.

2003, $n^{\circ} 3$ (julio) 
cada factor (Nunnally \& Bernstein, 1995; Martínez Arias, 1995; Kline, 2000). De este modo pudieron interpretarse 15 factores muy semejantes a los originalmente extraídos que explican un 60,17 de la varianza total de la prueba y se removieron 36 ítems. La mayoría de las escalas han modificado su denominación así como el número y contenido de los ítems respecto a las versiones anteriores del Cuestionario (Fogliatto, 1991; 1993).

\section{Confiabilidad}

Uno de los requisitos que debe reunir un instrumento psicométrico para su aplicación es que las mediciones obtenidas mediante su administración posean consistencia y estabilidad. El análisis de las dos dimensiones fundamentales de confiabilidad del CIP-R se describen a continuación.

\section{a) Estabilidad}

Para analizar la estabilidad del CIP-R se empleó la técnica test-retest que consiste en aplicar dos veces el inventario a una misma muestra y luego estimar la correlación entre los valores obtenidos en la primera y segunda administración. Se aplicó el instrumento a una muestra de 156 estudiantes secundarios (mujeres 68.8\%; varones 31.2\%; media de edad: 17.50), a comienzos del año lectivo y cuando los alumnos se encontraban a final de período (aproximadamente 6 meses después) volvieron a responder al inventario. Los coeficientes de confiabilidad obtenidos son satisfactorios para todas las escalas. La Tabla 1 presenta los coeficientes correspondientes a cada escala. 
Tabla 1 - Estabilidad de las escalas del CIP-R $(\mathrm{N}=154)$

\begin{tabular}{lc}
\hline Escalas de Intereses & Coeficientes de estabilidad (r) \\
\hline Cálculo & $\mathbf{. 9 0}$ \\
Científica & $\mathbf{8 0}$ \\
Diseño &. $\mathbf{7 8}$ \\
Tecnológica & $\mathbf{8 3}$ \\
Geoastronómica & $\mathbf{. 8 2}$ \\
Naturalista & $\mathbf{8 8}$ \\
Sanitaria & $\mathbf{. 8 9}$ \\
Asistencial & $\mathbf{7 9}$ \\
Jurídica & $\mathbf{. 8 4}$ \\
Económica & $\mathbf{8 8}$ \\
Comunicacional & $\mathbf{8 7}$ \\
Humanística & $\mathbf{. 8 5}$ \\
Artística & $\mathbf{. 8 4}$ \\
Musical & $\mathbf{8 5}$ \\
Lingüística & $\mathbf{8 2}$ \\
\hline
\end{tabular}

\section{b) Consistencia Interna}

Con la finalidad de estimar esta dimensión fundamental de confiabilidad la prueba fue administrada a 810 estudiantes de nivel polimodal (mujeres 57,1\%, varones 42,9\%) con un rango de edad entre 16 y 20 años (media 17,48) y que cursaban el último año del ciclo de especialización en sus distintas modalidades educativas (Producción de Bienes y Servicios, Arte y Comunicación, Diseño, Ciencias Sociales, Bachillerato Musical, Ciencias Naturales, Gestión y Administración de Empresas). Se empleó el coeficiente alfa de Cronbach, que es un indicador de la covarianza de los reactivos de una escala (Muñiz, 2001). Los resultados obtenidos (ver Tabla 2) revelan una consistencia interna adecuada de todas las escalas.

\begin{tabular}{lc} 
Tabla 2. Coeficiente Alfa de Cronbach de las escalas del CIP-R $(\mathrm{N}=810)$ \\
\hline Escalas de Intereses & Coeficiente alfa de Cronbach \\
\hline Cálculo & .90 \\
Científica & .86 \\
Diseño & .89 \\
Tecnológica & .90 \\
Geoastronómica & .83 \\
Naturalista & .89 \\
Sanitaria & .87 \\
Asistencial & .88 \\
Jurídica & .84 \\
Económica & .91 \\
Comunicacional & .84 \\
Humanística & .89 \\
Artística & .87 \\
Musical & .91 \\
Lingüística & .85 \\
\hline
\end{tabular}




\section{Validez}

La información obtenida acerca de la validez de una prueba es la más importante desde el punto de vista psicométrico y nos indica en que medida una prueba puede emplearse de un modo técnicamente y éticamente adecuado para alcanzar determinados propósitos . La validez es un concepto unitario y cada uno de los estudios que se detallan a continuación constituye una evidencia complementaria de validación de las escalas del CIP-R (Tornimbeni, Pérez, Baldo, Olaz y Fernández, 2003; APA, 1999).

\section{a) Evidencias de la estructura interna}

Como se describió en el apartado Construcción, el análisis de Componentes Principales y Rotación Varimax efectuado en una muestra representativa de estudiantes de ciclo polimodal $(\mathrm{N}=810)$ permitió obtener 15 factores que evidencian una estructura interna sólida y estable del Inventario. Otra evidencia importante en el caso de escalas obtenidas factorialmente es corroborar la relativa independencia entre las mismas mediante el análisis de sus intercorrelaciones. La Tabla 3 presenta los coeficientes de correlación entre las 15 escalas del CIP. Como puede observarse las correlaciones son bajas, excepto entre escalas teóricamente relacionadas donde se aprecian coeficientes moderados.

Tabla 3- Intercorrelaciones entre las escalas del CIP - R (r de Pearson,)

\begin{tabular}{|c|c|c|c|c|c|c|c|c|c|c|c|c|c|c|c|}
\hline Ítem & 1 & 2 & 3 & 4 & 5 & 6 & 7 & 8 & 9 & 10 & 11 & 12 & 13 & 14 & 15 \\
\hline \multicolumn{16}{|c|}{$\mathrm{N}=810$} \\
\hline 1 & - & .32 & .23 & .11 & .06 & -.03 & .19 & .11 & .16 & .07 & .19 & .23 & .18 & .03 & .00 \\
\hline 2 & & - & .16 & -.11 & .06 & .06 & .21 & $.41 *$ & .10 & -.02 & -.01 & $.44 *$ & .15 & .00 & .09 \\
\hline 3 & & & - & .03 & -.14 & .07 & $.43^{*}$ & $.37 *$ & .05 & -.15 & .28 & $.44 *$ & .13 & -.09 & -.11 \\
\hline 4 & & & & - & .21 & -.04 & .07 & -.09 & -.10 & $.38^{*}$ & $.36^{*}$ & .10 & -.03 & .03 & .24 \\
\hline 5 & & & & & - & .22 & -.22 & -.08 & .00 & $.38^{*}$ & -.10 & -.03 & $.31 *$ & $.42 *$ & $.61^{*}$ \\
\hline 6 & & & & & & - & .16 & .14 & $.31 *$ & .05 & -.06 & .07 & $.42 *$ & $.44^{*}$ & .26 \\
\hline 7 & & & & & & & - & $.40^{*}$ & $.33 *$ & -.10 & .29 & $.39 *$ & .10 & -.11 & -.11 \\
\hline 8 & & & & & & & & - & .16 & -.05 & -.01 & $.44 *$ & .12 & -.08 & .14 \\
\hline 9 & & & & & & & & & - & .05 & .08 & .12 & $.39 *$ & $.42 *$ & .04 \\
\hline 10 & & & & & & & & & & - & -.09 & -.14 & .09 & $.32 *$ & $.34 *$ \\
\hline 11 & & & & & & & & & & & - & .24 & .00 & -.10 & .01 \\
\hline 12 & & & & & & & & & & & & - & .20 & -.10 & .07 \\
\hline 13 & & & & & & & & & & & & & - & $.53 *$ & $.30 *$ \\
\hline 14 & & & & & & & & & & & & & & - & $.35 *$ \\
\hline 15 & & & & & & & & & & & & & & & - \\
\hline
\end{tabular}




\section{b) Evidencias de diferencias entre grupos contrastados}

Una modalidad para verificar la validez de las escalas con el procedimiento de comparación entre grupos contrastados es obtener las medias, desviaciones standards y puntuaciones " $\mathrm{t}$ " para el grupo de estudiantes secundarios de ambos sexos, respectivamente. El CIP-R fue administrado a una muestra de 810 estudiantes de nivel polimodal (mujeres 57,1\%, varones 42,9\%) con un rango de edad entre 16 y 20 años (media 17,48). Los resultados obtenidos (Tabla 4) son congruentes con lo teóricamente esperado, vale decir, los varones obtienen las medias más elevadas en áreas de intereses relacionadas con carreras predominantemente masculinas y las mujeres en las escalas asociadas con carreras tradicionalmente "femeninas" desde el punto de vista de la investigación de patrones de intereses diferenciados por sexo. Puede observarse en la Tabla 4 que los valores " $\mathrm{t}$ " son significativamente distintos de cero en las escalas Humanística, Tecnológica, Naturalista, Asistencial, Artística, Sanitaria, Cálculo, Jurídica, Comunicacional, Geoastronómica, Científica y Diseño. La muestra femenina obtiene puntuaciones significativamente más elevadas en las escalas Comunicacional, Jurídica, Humanística, Asistencial, Sanitaria y Artística. Por su parte, los varones alcanzaron valores medios significativamente superiores a las mujeres en las escalas Tecnológica, Naturalista, Cálculo, Geoastronómica, Ciencias Básicas y Diseño. No se presentan diferencias significativas en los valores medios obtenidos por ambos sexos en las escalas Lingüística, Musical y Económica. Estos resultados son congruentes con los obtenidos por estudios semejantes en la literatura de intereses (Holland, 1997).

Tabla 4 - Medias, Desviaciones Estándar y valores "t" por escala y sexo

\begin{tabular}{lcccccc}
\hline \multicolumn{1}{c}{ Escalas } & Media masc. & Media fem. & S masc. & S fem. & t & Sign. \\
\hline Lingüística & 11.24 & 11.61 & 3.61 & 3.84 & 1,40 & .16 \\
Musical & 16.30 & 15.77 & 5.60 & 5.37 & $-1,35$ & .18 \\
Humanística & 13.49 & 14.67 & 4.60 & 5.95 & 3.40 &. $\mathbf{0 0 2}$ \\
Económica & 18.66 & 18.25 & 6.30 & 5.95 & -.95 & .055 \\
Tecnológica & 16.02 & 10.90 & 4.76 & 3.25 & -18.1 & $\mathbf{. 0 0 1}$ \\
Naturalista & 15.11 & 13.54 & 4.88 & 4.40 & -4.77 & $\mathbf{. 0 0 1}$ \\
Asistencial & 14.23 & 17.52 & 4.62 & 4.48 & 10.15 & $\mathbf{. 0 0 1}$ \\
Artística & 13.79 & 16.22 & 4.52 & 4.45 & 7.62 & $\mathbf{. 0 0 1}$ \\
Sanitaria & 12.91 & 14.40 & 4.36 & 4.76 & 4.56 & $\mathbf{. 0 0 1}$ \\
Cálculo & 12.32 & 11.10 & 4.33 & 4.42 & -3.90 & $\mathbf{. 0 0 1}$ \\
Jurídica & 10.70 & 11.93 & 3.44 & 3.83 & 4.70 & $\mathbf{. 0 0 1}$ \\
Comunicacional & 15.92 & 16.97 & 4.45 & 4.33 & 3.34 & $\mathbf{. 0 0 1}$ \\
Geoastronómica & 11.88 & 11.31 & 3.46 & 3.49 & -2.30 & $\mathbf{. 0 2 2}$ \\
Diseño & 12.50 & 10.26 & 4.22 & 3.51 & -8.22 & $\mathbf{. 0 0 1}$ \\
Científica & 12.37 & 10.55 & 4.01 & 3.61 & -6.78 & $\mathbf{. 0 0 1}$ \\
\hline
\end{tabular}




\section{c)Evidencia de relaciones entre las escalas y el criterio elección de carrera}

La validez de criterio hace referencia a la precisión con que las puntuaciones de tests predicen el desempeño en criterios externos (APA, 1999). En el caso de las medidas de intereses, como ya se señaló, una de las principales utilidades es su capacidad predictiva con respecto a las variables elección de carrera e intenciones de elección de carrera (Lent, Brown \& Hackett, 1994; Holland, 1994). En este caso el CIP-R fue administrado a comienzos del período lectivo a una muestra de 834 estudiantes que cursaban el último año de su ciclo educativo polimodal en sus diferentes especializaciones $\mathrm{y}$, aproximadamente 6 meses después (cuando los estudiantes normalmente ya deciden que carrera continuar), los mismos estudiantes fueron entrevistados telefónicamente para conocer si habían realizado alguna elección de carrera. Se obtuvo una muestra de 434 estudiantes (mujeres $53.8 \%$, varones $46.2 \%$, media de edad 17.15) que informaron haber elegido una carrera superior. Se correlacionaron las puntuaciones obtenidas por cada sujeto en cada una de las escalas del Inventario versus todas sus puntuaciones en elección de carrera. La correlación empleada fue el coeficiente punto biserial (rpb) que es el adecuado cuando se comparan variables continuas (puntuaciones en las escalas) con variables dicotómicas (elección de carrera) (Nunnally \& Bernstein, 1995). La Tabla 5 presenta las correlaciones significativas con elecciones de carreras encontradas para cada escala. Puede observarse que todas las escalas evidencian utilidad predictiva y discriminativa; cada una de ellas se correlaciona significativamente con elecciones de carreras congruentes con el contenido de cada escala y no se correlaciona significativamente con elecciones en carreras teóricamente incongruentes. 
Tabla 5- Correlaciones significativas (rpb) entre cada escala y las elecciones de carrera (se omitieron las correlaciones inferiores a .10)

\begin{tabular}{|c|c|c|}
\hline Escalas del CIP-R & $\begin{array}{l}\text { Elecciones de } \\
\text { Carreras superiores }\end{array}$ & Correlación punto biserial \\
\hline \multirow[t]{2}{*}{ Cálculo } & Ingeniería en Sistemas & .22 \\
\hline & Contador Público & .29 \\
\hline Científica & Bioquímica & .23 \\
\hline Diseño & Arquitectura & .48 \\
\hline \multirow[t]{4}{*}{ Tecnológica } & Ingeniería en Sistemas & .33 \\
\hline & Ingeniería Electrónica & .25 \\
\hline & Ingeniería Mecánica & .19 \\
\hline & Educación Física & .10 \\
\hline Geoastronómica & Astronomía & .17 \\
\hline \multirow[t]{3}{*}{ Naturalista } & Ingeniería Agronómica & .29 \\
\hline & Veterinaria & .25 \\
\hline & Biología & .17 \\
\hline \multirow[t]{3}{*}{ Sanitaria } & Medicina & .52 \\
\hline & Odontología & .19 \\
\hline & Fisioterapia & .13 \\
\hline \multirow[t]{2}{*}{ Asistencial } & Psicología & .23 \\
\hline & Trabajo Social & .18 \\
\hline Jurídica & Abogacía & .51 \\
\hline \multirow[t]{4}{*}{ Económica } & Contador & .35 \\
\hline & Administración & .28 \\
\hline & Marketing & .14 \\
\hline & Abogacía & .12 \\
\hline \multirow[t]{2}{*}{ Comunicacional } & Periodismo & .25 \\
\hline & Cinematografía & .13 \\
\hline \multirow[t]{3}{*}{ Humanística } & Psicología & .21 \\
\hline & Periodismo & .14 \\
\hline & Historia & .12 \\
\hline \multirow[t]{3}{*}{ Artística } & Arquitectura & .18 \\
\hline & Pintura & .15 \\
\hline & Diseño Gráfico & .18 \\
\hline \multirow[t]{2}{*}{ Musical } & Música & .26 \\
\hline & Cinematografía & .13 \\
\hline \multirow[t]{2}{*}{ Lingüística } & Traductor en Inglés & .31 \\
\hline & Turismo & .11 \\
\hline
\end{tabular}

\section{Discusión}

Los objetivos del presente trabajo fueron revisar y actualizar el Cuestionario de Intereses Profesionales (Fogliatto, 1989; 1993). Con esta finalidad se realizó un nuevo análisis factorial que permitió replicar la misma estructura interna del test en su versión anterior, con 15 escalas primarias de intereses, y eliminar 36 reactivos del pool inicial. El nuevo inventario, que hemos denominado Cuestionario de Intereses Profesionales Revisado (CIP-R), fue analizado en sus dimensiones psicométricas esenciales: consistencia interna, estabilidad, validez conceptual y de criterio, con resultados 
satisfactorios para todas las escalas. El CIP-R se integra como un módulo específico a la nueva forma del Sistema de Orientación Vocacional Informatizado, SOVI 3 (Fogliatto \& Pérez, en prensa), un programa que contempla también evaluación de Autoeficacia para Inteligencias Múltiples (Pérez, 2001) y un módulo renovado de información para carreras con links a sitios académicos en la Web.

\section{Referencias}

American Psychological Association (APA) (1999). Standards for Psychological and Educational Tests. Washington, DC.

Barak, A. (1981). Vocational Interests: A cognitive view. Journal of Vocational Behavior. 19 (1).

Care, E. (1996). The structure of interests related to college course destinations. Journal of Career Assessment. 4, 77-89.

Cortada de Kohan, N. (1974). Problemas para la elaboración y tipificación de tests en distintos ambientes culturales. Revista de Psicología General y Aplicada. 29.

Cronbach, L. (1998). Fundamentos de los tests psicológicos. Madrid: Biblioteca Nueva.

Fogliatto, H. et al. (1973). Análisis de instrumentos utilizados en orientación vocacional. Facultad de Filosofía y Humanidades. Universidad Nacional de Córdoba.

Fogliatto, H. et al.(1980). Los cuestionarios de intereses. Un análisis crítico. Revista Latinoamericana de Psicología. XII, 503-512.

Fogliatto, H. (1991). Cuestionario de intereses profesionales. Manual. Buenos Aires: Guadalupe.

Fogliatto, H. (1993). Cuestionario de Intereses Profesionales Computarizado (CIPC). Manual y Diskette. Buenos Aires: Guadalupe.

Fogliatto, H. \& Pérez, E. (1997). Sistema de orientación vocacional informatizado (SOVI). Manual y diskette. Buenos Aires: Guadalupe.

Fogliatto, H. \& Pérez, E. (2001). SOVI 2001. Manual y CD-ROOM. Buenos Aires: Guadalupe

Fogliatto, H. \& Pérez, E. (en prensa). Sistema de Orientación Vocacional Informatizado. SOVI 3. Manual. Buenos Aires: Paidós 
Furnham, A. (1992). Personalidad y diferencias individuales en el trabajo. Madrid: Pirámide.

Hogan, R. (1983). A socioanalityc theory of personality. Lincoln, NE: University of Nebraska Press.

Holland, J. (1994). Self-Directed Search. Professional Manual. Odessa, FL: Psychological Assessement Resources.

Holland, J. (1997). Making Vocational Choices. Englewood Cliffs, NJ: Prentice-Hall.

Hood, A. \& Johnson, R. (2002). Assessment in Counseling (Third edition). Alexandría (VA): American Counseling Association

Kline, P. (2000). Handbook of Psychological Tests. London: Rotledge

Lent, R.; Brown, D; Hackett,G. (1994) Toward a Unifying Social Cognitive Theory of Career and Academic Interest, Choice, and Performance. Journal of Vocational Behavior.45, 79-122.

Martínez Arias, R. (1995). Psicometría. Madrid: Síntesis Psicológica.

Muñiz, J. (2001). Teoría Clásica de los Tests. Madrid: Pirámide.

Nunnally, J. \& Bernstein, I. (1995). Teoría psicométrica. México: Mc Graw Hill.

Pérez, E. (2001). Construcción de un Inventario Informatizado de Autoeficacia para Inteligencias Múltiples. Tesis Doctoral (Inédita). Córdoba: Facultad de Psicología, Universidad Nacional de Córdoba

Rivas, F. (1988). Psicología Vocacional. Enfoques del Asesoramiento. Madrid: Morata.

Roe, A. (1972). Psicología de las ocupaciones. Madrid: Marova.

Schneider, P. et al (1996). Examining the relation between Holland's RIASEC Model and the Interpersonal Circle. Measurement and Evaluation in Counseling and Development. 29, 123-133.

Strong, E. (1951). Vocational interests 18 years after college. Minneapolis: University of Minnesota.

Super, D. (1967). Psicología de los intereses y las vocaciones. Buenos Aires: Kapeluz.

Tetreau, B. \& Trahan, M. (1986). Test Visuel d'Interets Tetreau-Trahan. Manuel d'usage. Montreal: SECOROP.

Tornimbeni, S., Pérez, E., Baldo, M., Olaz, F. \& Fernández, A. (2003). Introducción a los tests psicológicos (Segunda edición). Córdoba: Brujas.

Evaluar.

2003, $n^{\circ} 3$ (julio) 
Watkins, C. (1994). The practice of vocational assessment by counseling psychologists. The Counseling Psychologists. 22, 115-128. 


\section{Anexo}

\section{Cuestionario de Intereses Profesionales Revisado CIP-R}

\section{Instrucciones:}

El Cuestionario de Intereses Profesionales Revisado no es un examen de conocimientos, por lo tanto no contiene respuestas correctas o incorrectas; es un registro de tus intereses vocacionales, y toda respuesta es considerada satisfactoria si es verdaderamente representativa de tus preferencias. Es por ello que la tarea debe realizarse de manera personal. Cada uno de los ítems representa una actividad propia de trabajos profesionales o de tareas realizadas por los estudiantes durante el aprendizaje de una carrera. Examina cada ítem e indica con una cruz en el casillero correspondiente, tu Desagrado (D), Indiferencia (I), o Agrado (A) por el mismo.

Por ejemplo:

Ilustrar cuentos infantiles

La respuesta del ejemplo indica el agrado de la persona por este ítem.

Recuerda que debes responder a todos los ítems dando solo una alternativa de respuesta para cada ítem.

Si no comprendes el significado de alguna palabra o ítem consulta con quien te administra el cuestionario para que puedas dar una respuesta segura.

Datos personales

\begin{tabular}{|l|l|l|l|l|l|}
\hline Nombre & Sexo: & M & F & Edad: \\
\hline Domicilio & E-Mail \\
\hline Teléfono &
\end{tabular}

(nivel Polimodal)

Indica con una X la modalidad de especialización de tu Colegio

1. Ciencias Sociales

2. Producción de bienes y servicios

3. Gestión y Administración de empresas

4. Ciencias Naturales

5. Arte, diseño y comunicación

6. Otra: (Especificar) 


\section{Hoja de Respuestas}






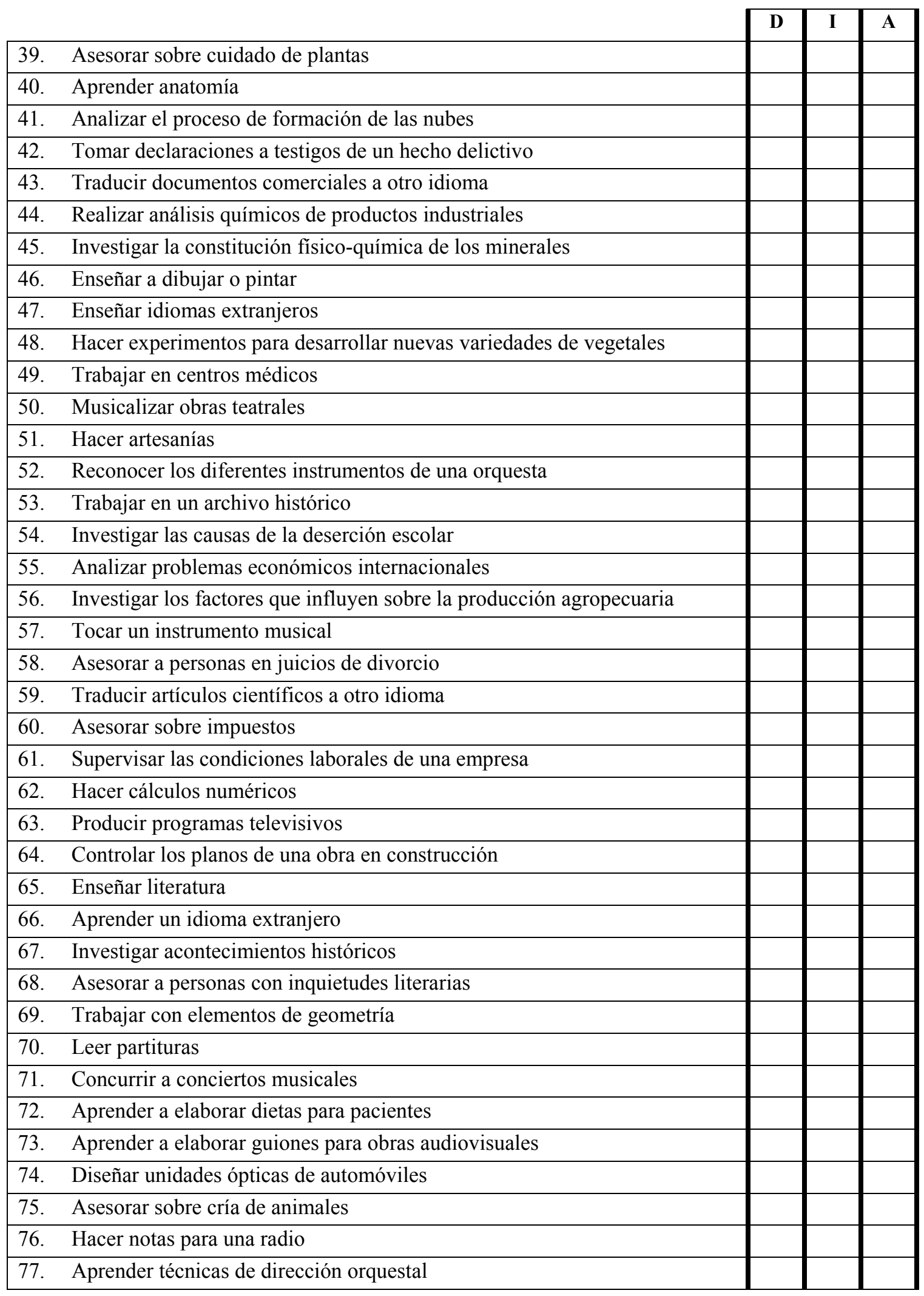

Evaluar.

2003, $n^{\circ} 3$ (julio) 




